\title{
Delay Guarantee versus Throughput in Broadcast Fading Channels
}

\author{
Masoud Sharif and Babak Hassibi ${ }^{1}$ \\ Dept. Electrical Engineering \\ California Institute of Technology, Pasadena, CA 91125 \\ \{masoud, hassibi\}@systems. caltech.edu
}

\begin{abstract}
We consider a single-antenna broadcast fading channel with $n$ backlogged users. Assuming the transmission is packet-based, we define the delay as the minimum number of channel uses that guarantees all $n$ users successfully receive $m$ packets. A delay optimal strategy such as round-robin achieves the delay of $m n$. For the optimal throughput strategy (i.e. transmitting to the user with the best channel condition at each channel use), we derive the mean and variance of the delay for any $m$ and $n$. For large $n$, it is proved that the expected delay in receiving the first packet in all users scales like $n \log n$ as opposed to $n$ for the round-robin scheduling.
\end{abstract}

\section{EXTENDED SUmmaRY}

Resource allocation in wireless systems is aiming for two conflicting goals, firstly providing quality of service such as delay and fairness to users, and secondly maximizing the throughput of the system [1]. In order to maximize the throughput, the resource should be scheduled to the user with the highest capacity or with the best channel condition. Clearly this policy will cause non-fair scheduling and delay in sending packets.

In this paper, we consider a single antenna broadcast channel with $n$ receivers. Our channel model is a block Rayleigh fading channel which changes independently from one block to the other. The transmission is packet based and therefore at each channel use, the transmitter sends one packet at each channel use. In the transmitter, we assume there are $n$ queues corresponding to each user and that there is always a packet to be sent to each user.

We define the delay $D_{m, n}$ as the number of channel uses required to guarantee that all the users will receive $m$ packets successfully. Of course, the delay is a function of the number of users $n$, and the number of packets $m$. A delay-optimal strategy is round-robin scheduling which clearly achieves the optimal delay of $m n$, however, round-robin scheduling is not throughput optimal. Throughput optimal strategies, on the other hand, will have to contend with delay hits. The overriding question in this paper is to characterize the delay characteristics for the throughput optimal scheduling in broadcast fading channels.

Theorem 1. Suppose we have $n$ users such that the probability of choosing the $i$ 'th user is $\frac{a_{i}}{n}$ and the probability of dropping a packet is $P_{e}$. Defining $S_{m}(t)=\sum_{k=0}^{m-1} \frac{t^{k}}{k !}$, then

$$
E\left(D_{m, n}\right)=(n+1) \int_{0}^{\infty}\left(1-\prod_{i=1}^{n}\left(1-S_{m}\left(\beta_{i} t\right) e^{-\beta_{i} t}\right)\right) d t
$$

\footnotetext{
${ }^{1}$ This work was supported in part by NSF Grant CCR-0133818, by the office of Naval Research Grant N00014-02-1-0578, and By Caltech's Lee Center for Advanced Networking.
}

$$
\begin{aligned}
& \text { where } \beta_{i}=\left(1-P_{e}\right) a_{i} \text {. Furthermore, } \\
& \qquad \begin{array}{c}
\sigma^{2}\left(D_{m, n}\right)=2(n+1)^{2} \int_{0}^{\infty} t\left(1-\prod_{i=1}^{n}\left(1-S_{m}\left(\beta_{i} t\right) e^{-\beta_{i} t}\right)\right) d t \\
-E\left(D_{m, n}\right)-\left(E\left(D_{m, n}\right)\right)^{2} .
\end{array}
\end{aligned}
$$

The Theorem extends a result of Newman and Shepp [2]. Assuming a homogeneous network, we can state the following asymptotic results for the delay.

Theorem 2. Consider the setting of Theorem 1,

1. For $m$ fixed and $n \rightarrow \infty$, we have

$$
\begin{aligned}
E\left(D_{m, n}\right) & =\frac{1}{1-P_{e}} n \log n+n(m-1) \log \log n+O(n), \\
\sigma^{2}\left(D_{m, n}\right) & =O\left(n^{2}\right) .
\end{aligned}
$$

2. For $m=\log n$ and $n \rightarrow \infty$, we have

$$
E\left(D_{m, n}\right)=\frac{3.146}{1-P_{e}} n \log n+O(n \log \log n) .
$$

3. For $m=(\log n)^{r}$ where $r>1$ is fixed and $n \rightarrow \infty$, then

$$
E\left(D_{m, n}\right)=\frac{1}{1-P_{e}} n(\log n)^{r}+O(n \log n)=m n+O(n \log n) .
$$

4. For $n$ fixed and $m \rightarrow \infty$,

$$
E\left(D_{m, n}\right)=\frac{1}{1-P_{e}} m n+O(n \log m) .
$$

Therefore the effect of opportunistic transmission to the best user at each time on delay is an increase by a multiplicative factor of $\log n$ for fixed $m$. We can in fact improve this factor of $\log n$ by sacrificing on rate [3].

A generalization is the case where we have an $M$-antenna transmitter which at each channel use, transmits to $M$ different users. We can prove that for $M$ not growing faster than $\log n$, the expected delay in transmitting the first packet to all users (denoted by $D_{1, n}^{M}$ ) for a homogeneous network is

$$
E\left(D_{1, n}^{M}\right)=\frac{1}{1-P_{e}} \frac{n \log n}{M+O\left(M^{2} / n\right)}+O(n \log \log n) .
$$

Therefore, multiple transmit antenna transmitter incurs pretty much the same delay as that of a single antenna systems that transmit $M$ times faster. Although the gain on delay in using multiple transmit antennas is not that much, multiple transmit antenna can significantly improve the long term fairness in a heterogeneous network [3].

\section{REFERENCES}

[1] R. A. Berry and R. G. Gallager, "Communication over fading channels with delay constraints," IEEE Trans. Info., vol. 48, no. 5, pp. 1135-1149, May 2002.

[2] D. J. Newman and L. Shepp, "The double dixie cup problem," Amer. Math. Monthly, vol. 67, no. 1, pp. 58-61, Jan. 1960.

[3] M. Sharif and B. Hassibi, "Delay analysis of the throughput optimal scheduling in broadcast fading channels," in preparation. 